\title{
Bimodules in Group Graded Rings
}

\author{
Johan Öinert ${ }^{1}$ (1)
}

Received: 10 January 2017 / Accepted: 20 April 2017 / Published online: 10 May 2017

(C) The Author(s) 2017. This article is an open access publication

\begin{abstract}
In this article we introduce the notion of a controlled group graded ring. Let $G$ be a group, with identity element $e$, and let $R=\oplus_{g \in G} R_{g}$ be a unital $G$-graded ring. We say that $R$ is $G$-controlled if there is a one-to-one correspondence between subsets of the group $G$ and (mutually non-isomorphic) $R_{e}$-sub-bimodules of $R$, given by $G \supseteq H \mapsto \oplus_{h \in H} R_{h}$. For strongly $G$-graded rings, the property of being $G$-controlled is stronger than that of being simple. We provide necessary and sufficient conditions for a general $G$-graded ring to be $G$-controlled. We also give a characterization of strongly $G$-graded rings which are $G$-controlled. As an application of our main results we give a description of all intermediate subrings $T$ with $R_{e} \subseteq T \subseteq R$ of a $G$-controlled strongly $G$-graded ring $R$. Our results generalize results for artinian skew group rings which were shown by Azumaya 70 years ago. In the special case of skew group rings we obtain an algebraic analogue of a recent result by Cameron and Smith on bimodules in crossed products of von Neumann algebras.
\end{abstract}

Keywords Graded ring · Strongly graded ring · Crossed product · Skew group ring · Bimodule $\cdot$ Picard group

Mathematics Subject Classification (2010) $16 \mathrm{~S} 35 \cdot 16 \mathrm{~W} 50 \cdot 16 \mathrm{D} 40$

\section{Introduction}

Recently, Cameron and Smith [2] studied bimodules over a von Neumann algebra $M$ in the context of an inclusion $M \subseteq M \rtimes_{\alpha} G$, where $G$ is a group acting on $M$ by *-automorphisms

Presented by Kenneth Goodearl.

Johan Öinert

johan.oinert@bth.se

1 Department of Mathematics and Natural Sciences, Blekinge Institute of Technology,

37179 Karlskrona, Sweden 
and $M \rtimes_{\alpha} G$ is the corresponding crossed product von Neumann algebra. They have shown [2, Theorem 4.4(i)] that if $G$ is a discrete group acting by outer $*$-automorphisms on a simple ${ }^{1}$ von Neumann algebra $M$, then there is a bijective correspondence between subsets of $G$ and $B$-closed (i.e. closed in the Bures-topology) $M$-sub-bimodules of $M \rtimes_{\alpha} G$.

It is natural to ask whether the same correspondence holds for a skew group ring, which is the algebraic analogue of a crossed product von Neumann algebra. To be more precise, if $G$ is a group which is acting by outer automorphisms on a simple and unital ring $A$, then we ask whether each $A$-sub-bimodule of the skew group ring $A \rtimes_{\alpha} G$ is of the form $\oplus_{h \in H} A u_{h}$ for some subset $H \subseteq G$, where $\varnothing$ corresponds to the zero-module. As it turns out, although it was not the main focus of his investigation, in one of his proofs Azumaya has observed this correspondence in the case when $G$ is finite [1].

The purpose of this article is to study in a systematic way the same type of correspondence in the more general context of group graded rings.

Throughout this article, let $R$ be an associative and unital ring and let $G$ be a multiplicatively written group with identity element $e \in G$. For subsets $X$ and $Y$ of $R$, we let $X Y$ denote the set of all finite sums of elements of the form $x y$, for $x \in X$ and $y \in Y$. If there is a family $\left\{R_{g}\right\}_{g \in G}$ of additive subgroups of $R$ such that

$$
R=\oplus_{g \in G} R_{g} \quad \text { and } \quad R_{g} R_{h} \subseteq R_{g h}
$$

for all $g, h \in G$, then the ring $R$ is said to be $G$-graded (or graded by $G$ ). A $G$-graded ring $R$ for which $R_{g} R_{h}=R_{g h}$ holds, for all $g, h \in G$, is said to be strongly $G$-graded.

If $R$ is a $G$-graded ring, then one immediately observes that $R_{e}$ is a subring of $R$ and that $1_{R} \in R_{e}$ (see e.g. [4, Proposition 1.4]). For any $g \in G, R_{g}$ is an $R_{e}$-bimodule. If $R$ is strongly $G$-graded, then for each $g \in G, R_{g}$ is finitely generated and projective as a left (right) $R_{e}$-module (see [6, Proposition 1.10]). Any $G$-graded ring $R$ will be viewed as an $R_{e}$-bimodule with scalar multiplication given by the ring multiplication in $R$. The set of all $R_{e}$-sub-bimodules of $R$ will be denoted by $\operatorname{Mod}_{R}\left(R_{e}\right)$. If $M, N \in \operatorname{Mod}_{R}\left(R_{e}\right)$ are isomorphic as $R_{e}$-bimodules, then we will write $M \cong N$.

Each subset of $G$ gives rise to an $R_{e}$-sub-bimodule of $R$. Indeed, if $H$ is a subset of $G$, then $R_{H}=\oplus_{h \in H} R_{h}$ is an $R_{e}$-sub-bimodule of $R$. We let the empty set give rise to the zero-module, i.e. $R_{\varnothing}=\{0\}$. It is natural to ask the following question:

When does every $R_{e}$-sub-bimodule of $R$ arise in this way?

We make the following definition.

Definition 1 ( $G$-controlled ring) A ring $R$ is said to be $G$-controlled if it is equipped with a $G$-gradation such that the following two assertions hold:

1. The map

$$
\varphi: \mathcal{P}(G) \rightarrow \operatorname{Mod}_{R}\left(R_{e}\right), \quad H \longmapsto \oplus_{h \in H} R_{h}
$$

is a bijection.

2. For $S, T \in \mathcal{P}(G), \varphi(S) \cong \varphi(T)$ if and only if $S=T$.

This article is organized as follows.

In Section 2 we recall important notions which will be used in subsequent sections. In Section 3 we give a complete characterization of $G$-controlled rings (see Theorem 1 ). We

\footnotetext{
${ }^{1}$ In fact their result is more general. They only assume that $M$ is a factor.
} 
also provide examples of $G$-controlled rings which are not strongly $G$-graded (see Examples 1 and 2). In Section 4 we first point out that $G$-controlled rings are often strongly $G$-graded (see Proposition 3 and Remark 1). We then give a characterization of strongly $G$-graded rings which are $G$-controlled (see Theorem 2). We also specialize this result to $G$-crossed products (incl. skew group rings) to see how $G$-controlness is connected to outerness (see Corollary 2 and Remark 2). This shows how our results generalize those of Azumaya [1]. In Section 5 we give a description of all intermediate subrings $T$ where $R_{e} \subseteq T \subseteq R$ of a strongly $G$-graded and $G$-controlled ring $R$ (see Proposition 4). In Section 6 we present some simplicity results on strongly $G$-graded rings and explain how they are related to our investigation of $G$-controlled rings. We also present some open questions (see Questions 1, 2 and 3).

\section{Preliminaries and Notation}

The centralizer of a non-empty subset $S$ of a ring $T$ will be denoted by $C_{T}(S)$ and is defined as the set of all elements of $T$ that commute with each element of $S$. The center of $T$ is defined as $C_{T}(T)$ and will be denoted by $Z(T)$. The group of multiplication invertible elements of $T$ will be denoted by $U(T)$.

Let $R=\oplus_{g \in G} R_{g}$ be a $G$-graded ring. Each element $x \in R$ may be written as $x=$ $\sum_{g \in G} x_{g}$ where $x_{g} \in R_{g}$ is unique for each $g \in G$, and zero for all but finitely many $g \in G$. For $g \in G$ we define a map

$$
E_{g}: R \rightarrow R_{g}, \quad x=\sum_{h \in G} x_{h} \mapsto x_{g} .
$$

Notice that $E_{g}$ is an $R_{e}$-bimodule homomorphism. The support of $r \in R$ is defined as $\operatorname{Supp}(r)=\left\{g \in G \mid E_{g}(r) \neq 0\right\}$. An ideal $I$ of a $G$-graded ring $R$ is said to be graded if $I=\oplus_{g \in G}\left(I \cap R_{g}\right)$ holds. The ring $R$ is said to be graded simple if $R$ and $\{0\}$ are the only two graded ideals of $R$.

Recall that $R=\oplus_{g \in G} R_{g}$ is said to be a $G$-crossed product if $E_{g}(R) \cap U(R) \neq \varnothing$, for each $g \in G$. In that case, we may choose an invertible element $u_{g} \in R_{g}$, for each $g \in G$. Pick $u_{e}=1_{R}$. It is clear that $R_{g}=R_{e} u_{g}=u_{g} R_{e}$ and that the set $\left\{u_{g} \mid g \in G\right\}$ is a basis for $R$ as a left (and right) $R_{e}$-module. We now define two maps:

$$
\sigma: G \rightarrow \operatorname{Aut}\left(R_{e}\right) \text { by } \sigma_{g}(a)=u_{g} a u_{g}^{-1} \text { for } g \in G, a \in R_{e},
$$

and

$$
\alpha: G \times G \rightarrow U\left(R_{e}\right) \text { by } \alpha(g, h)=u_{g} u_{h} u_{g h}^{-1} \text { for } g, h \in G .
$$

One may now show that the following holds for any $g, h, t \in G$ and $a \in R_{e}$ (see e.g. [8, Proposition 1.4.2]):

$$
\begin{aligned}
& \text { 1. } \sigma_{g}\left(\sigma_{h}(a)\right)=\alpha(g, h) \sigma_{g h}(a) \alpha(g, h)^{-1} \\
& \text { 2. } \alpha(g, h) \alpha(g h, t)=\sigma_{g}(\alpha(h, t)) \alpha(g, h t) \\
& \text { 3. } \alpha(g, e)=\alpha(e, g)=1_{R} .
\end{aligned}
$$

Any two homogeneous elements $a \in R_{g}$ and $b \in R_{h}$ may be expressed as $a=a_{1} u_{g}$ and $b=b_{1} u_{h}$, for some $a_{1}, b_{1} \in R_{e}$, and their product is

$$
\begin{aligned}
a b=\left(a_{1} u_{g}\right)\left(b_{1} u_{h}\right)=a_{1}\left(u_{g} b_{1} u_{g}^{-1}\right) u_{g} u_{h} & =a_{1}\left(u_{g} b_{1} u_{g}^{-1}\right)\left(u_{g} u_{h} u_{g h}^{-1}\right) u_{g h} \\
& =a_{1} \sigma_{g}\left(b_{1}\right) \alpha(g, h) u_{g h} .
\end{aligned}
$$

Important examples of $G$-crossed products are given by e.g. skew group rings, twisted group rings and group rings. It is not difficult to see that $G$-crossed products are necessarily 
strongly $G$-graded. However, as e.g. Example 5 demonstrates, not all strongly $G$-graded rings are $G$-crossed products.

\section{A Characterization of $G$-Controlled Rings}

In this section we give a characterization of $G$-controlled rings (see Theorem 1). We begin by finding necessary conditions for a $G$-graded ring to be $G$-controlled.

Proposition 1 Let $G$ be a group and let $R$ be a G-graded ring. If $R$ is $G$-controlled, then the following five assertions hold:

(i) $R_{g} \cong R_{h}$ if and only if $g=h$ (where $g, h \in G$ );

(ii) $R_{g}$ is a (non-zero) simple $R_{e}$-sub-bimodule of $R$, for each $g \in G$;

(iii) $R_{e}$ is a simple ring;

(iv) $C_{R}\left(R_{e}\right)=Z\left(R_{e}\right)$;

(v) Every ideal of $R$ is graded.

Proof Let $R$ be a $G$-controlled ring, and let $\varphi$ be defined as in Definition 1 .

(i): Take $g, h \in G$. Notice that $\varphi(\{g\}) \cong \varphi(\{h\})$ if and only if $g=h$, i.e. $R_{g} \cong R_{h}$ if and only if $g=h$.

(ii): Take $g \in G$. By the injectivity of $\varphi$ we get that $R_{g} \neq\{0\}$, and by the surjectivity of $\varphi, R_{g}$ can not contain any proper non-zero $R_{e}$-sub-bimodule. Thus, $R_{g}$ is a simple $R_{e}$-subbimodule of $R$.

(iii): This follows immediately from (ii).

(iv): Notice that $C_{R}\left(R_{e}\right)$ is a $G$-graded subring of $R$. Take $g \in G$ and let $x_{g} \in$ $C_{R}\left(R_{e}\right) \cap R_{g}$ be non-zero. Define $f: R_{e} \rightarrow R_{g}, r \mapsto r x_{g}$. Clearly, $f$ is an $R_{e}$-bimodule homomorphism. Using (ii) we conclude that ker $f=\{0\}$ and that $\operatorname{im} f=R_{g}$, i.e. $f$ is an isomorphism. From (i) we get $g=e$. Hence, $C_{R}\left(R_{e}\right) \subseteq R_{e}$ which yields $C_{R}\left(R_{e}\right)=Z\left(R_{e}\right)$.

(v): Every ideal $I$ of $R$ is an $R_{e}$-sub-bimodule of $R$. Hence $I=\oplus_{h \in H} R_{h}$ for some subset $H \subseteq G$. In particular, $I$ is graded.

We now begin our search for sufficient conditions for $G$-controlness by showing the following essential lemma.

Lemma 1 Let $S$ be a unital ring and let $M$ and $N$ be simple $S$-bimodules which are non-isomorphic. For any $x \in M \backslash\{0\}$ and $y \in N \backslash\{0\}$ there is some $n \in \mathbb{Z}_{+}$and $s_{1}^{(1)}, \ldots, s_{n}^{(1)}, s_{1}^{(2)}, \ldots, s_{n}^{(2)} \in S$ such that $\sum_{i=1}^{n} s_{i}^{(1)} x s_{i}^{(2)} \neq 0$ and $\sum_{i=1}^{n} s_{i}^{(1)} y s_{i}^{(2)}=0$.

Proof Take $x \in M \backslash\{0\}$ and $y \in N \backslash\{0\}$. We notice that $S x S=M$ and $S y S=N$. Seeking a contradiction, suppose that $\sum_{i=1}^{n} s_{i}^{(1)} x s_{i}^{(2)}=0$ whenever $\sum_{i=1}^{n} s_{i}^{(1)} y s_{i}^{(2)}=0$. We define a map $f: N \rightarrow M, \quad \sum_{i=1}^{n} s_{i}^{(1)} y s_{i}^{(2)} \mapsto \sum_{i=1}^{n} s_{i}^{(1)} x s_{i}^{(2)}$. By our assumption $f$ is a welldefined homomorphism of $S$-bimodules. Moreover, by the unitality of $S$ and the simplicity of $M$ and $N$ we conclude that $f$ is an isomorphism. This is a contradiction.

Proposition 2 Let $G$ be a group and let $R=\oplus_{g \in G} R_{g}$ be a G-graded ring. Suppose that $R_{g}$ is a simple $R_{e}$-sub-bimodule of $R$, for each $g \in G$, and that $R_{g} \cong R_{h}$ if and only if $g=h$, for $g, h \in G$. If $P$ is a non-zero $R_{e}$-sub-bimodule of $R$ and $x \in P \backslash\{0\}$, then $R_{g}$ is an $R_{e}$-sub-bimodule of $P$, for each $g \in \operatorname{Supp}(x)$. In particular, $P=\oplus_{s \in S} R_{s}$ for some subset $S \subseteq G$. 
Proof Take $x \in P \backslash\{0\}$ and $g \in \operatorname{Supp}(x)$. Choose $y \in P \backslash\{0\}$ such that $|\operatorname{Supp}(y)|$ is minimal amongst all elements satisfying $g \in \operatorname{Supp}(y) \subseteq \operatorname{Supp}(x)$. Seeking a contradiction, suppose that $|\operatorname{Supp}(y)|>1$. Choose some $h \in \operatorname{Supp}(y) \backslash\{g\}$. Using Lemma 1, with $S=R_{e}, M=$ $R_{g}$ and $N=R_{h}$, we conclude that there is some $y^{\prime} \in P$ such that $\left|\operatorname{Supp}\left(y^{\prime}\right)\right|<|\operatorname{Supp}(y)|$ and $g \in \operatorname{Supp}\left(y^{\prime}\right) \subseteq \operatorname{Supp}(x)$. This is a contradiction. Hence, $P \cap R_{g} \neq\{0\}$. Using that $R_{g}$ is a simple $R_{e}$-sub-bimodule of $R$ we conclude that $R_{g}$ is an $R_{e}$-sub-bimodule of $P$. From this it follows that $P=\oplus_{s \in S} R_{S}$ for some subset $S \subseteq G$.

Lemma 2 Let $G$ be a group and let $R$ be a $G$-graded ring such that $R_{g}$ is a simple $R_{e}$-subbimodule of $R$, for each $g \in G$. The following two assertions are equivalent:

(i) $R_{S} \cong R_{T}$ if and only if $S=T$ (where $S, T \subseteq G$ );

(ii) $R_{g} \cong R_{h}$ if and only if $g=h$ (where $g, h \in G$ ).

Proof (i) $\Rightarrow$ (ii): This is trivial.

(ii) $\Rightarrow$ (i): The "if" statement is trivial. Now we show the "only if" statement. Suppose that $f: R_{S} \rightarrow R_{T}$ is an $R_{e}$-bimodule isomorphism. Take $s \in S$. Then $f\left(R_{S}\right)$ is a simple $R_{e^{-}}$ sub-bimodule of $R_{T}$. By Proposition 2 we conclude that $f\left(R_{s}\right)=R_{t}$ for some $t \in T$. This shows that $R_{s} \cong R_{t}$ and by (ii) we get $s=t$. Thus, $s \in T$. Using that $s$ was chosen arbitrarily, we get $S \subseteq T$. In the same way we can show that $T \subseteq S$. This shows that $S=T$.

We are now ready to prove the first main result of this article.

Theorem 1 Let $G$ be a group and let $R$ be a $G$-graded ring. Then $R$ is $G$-controlled if and only if (a) $R_{g}$ is a simple $R_{e}$-sub-bimodule of $R$, for each $g \in G$; and (b) $R_{g} \cong R_{h}$ if and only if $g=h$, for $g, h \in G$.

Proof The "only if" statement follows from Proposition 1.

We now show the "if" statement. Suppose that (a) and (b) hold. By using the maps $E_{g}$, for $g \in G$, we may conclude that $\varphi$ (in Definition 1) is injective. By Proposition 2, $\varphi$ is surjective. Hence, $\varphi$ is a bijection. By Lemma 2 we get that assertion (2) of Definition 1 holds. This shows that $R$ is $G$-controlled.

We shall now present two examples of $G$-graded rings which are $G$-controlled but not strongly $G$-graded. In the first example the group $G$ is finite, and in the second example $G$ is infinite. Notice that these rings are not simple (cf. Proposition 3).

Example 1 Let $A$ be a simple unital ring. Choose a simple $A$-bimodule $M$ which is not isomorphic to $A$ as an $A$-bimodule. The $A$-bimodule $R=A \times M$ may be equipped with a multiplication defined by $(a, m)(b, n)=(a b, a n+m b)$. It is readily verified that $R$ is an associative and unital ring. Choose $G=C_{2}=\{e, g\}$, the group with two elements. Clearly, $R_{e}=A \times\{0\}$ and $R_{g}=\{0\} \times M$ defines a $G$-gradation on $R$.

As a concrete example, we may take $\beta$ to be an outer automorphism on $A$. Then we can take $M=A$ with bimodule structure given by $a \cdot m \cdot b=a m \beta(b)$. Clearly, $M$ is simple as an $A$-bimodule. We claim that $A \nsubseteq M$. Seeking a contradiction, suppose that $f: A \rightarrow M$ is an isomorphism of $A$-bimodules. Then there is some $v \in A$ such that $f(v)=1$. If we put $u=f(1)$, then we see that $v u=v \cdot u=v \cdot f(1)=f(v 1)=f(v)=1=f(v)=$ $f(1 v)=f(1) \cdot v=u \cdot v=u \beta(v)$. Hence, $u$ is invertible and for every $a \in A$ we have $a u=f(a)=u \cdot a=u \beta(a)$, i.e. $\beta(a)=u^{-1} a u$. Thus, $\beta$ is not outer. This is a contradiction. 
Example 2 Consider the first Weyl algebra $\mathcal{A}_{1}=\mathbb{C}\langle x, y\rangle /(y x-x y-1)$. Recall that $\mathcal{A}_{1}$ is a simple noetherian domain. Take any automorphism $\gamma: \mathcal{A}_{1} \rightarrow \mathcal{A}_{1}$ satisfying $\left\{a \in \mathcal{A}_{1} \mid \gamma^{n}(a)=a\right.$ for some $\left.n \neq 0\right\}=\mathbb{C}$. (We may e.g. choose $\gamma$ defined by $x \mapsto x-1$ and $y \mapsto y+1$.) Let us now define a free left $\mathcal{A}_{1}$-module $R=\oplus_{n \in \mathbb{Z}} \mathcal{A}_{1} u_{n}$ with basis $\left\{u_{n}\right\}_{n \in \mathbb{Z}}$. We define a multiplication on $R$ by

$$
a u_{n} \cdot b u_{m}= \begin{cases}a \gamma^{n}(b) u_{n+m} & \text { if } n=0 \text { or } m=0 \\ 0 & \text { otherwise. }\end{cases}
$$

It is not difficult to verify that this turns $R$ into a unital and associative ring which is $\mathbb{Z}$ graded, but not strongly $\mathbb{Z}$-graded. Moreover, $R_{n}=\mathcal{A}_{1} u_{n}$ is a simple $R_{0}$-sub-bimodule of $R$, for each $n \in \mathbb{Z}$. Take $n, m \in \mathbb{Z}$ with $n \neq m$. We claim that $R_{n}$ and $R_{m}$ can not be isomorphic as $R_{0}$-bimodules. Seeking a contradiction, suppose that there is an $R_{0}$-bimodule isomorphism $f: R_{n}=\mathcal{A}_{1} u_{n} \rightarrow R_{m}=\mathcal{A}_{1} u_{m}$. Then there is some non-zero $c \in \mathcal{A}_{1}$ such that $f\left(1 u_{n}\right)=c u_{m}$. But then $c^{2} u_{m}=c\left(c u_{m}\right)=c f\left(u_{n}\right)=f\left(c u_{n}\right)=f\left(u_{n} \gamma^{-n}(c)\right)=$ $f\left(u_{n}\right) \gamma^{-n}(c)=c u_{m} \gamma^{-n}(c)=c \gamma^{m-n}(c) u_{m}$. Using that $R_{m}$ is a free left $\mathcal{A}_{1}$-module, we get $c^{2}=c \gamma^{m-n}(c)$. By our assumptions we conclude that $c \in \mathbb{C}=Z\left(\mathcal{A}_{1}\right)$. Now, take any $b \in \mathcal{A}_{1} \backslash \mathbb{C}$. Then, we get

$$
c \gamma^{m}(b) u_{m}=c u_{m} b=f\left(u_{n}\right) b=f\left(u_{n} b\right)=f\left(\gamma^{n}(b) u_{n}\right)=\gamma^{n}(b) f\left(u_{n}\right)=\gamma^{n}(b) c u_{m}
$$

and hence $c \gamma^{m}(b)=\gamma^{n}(b) c=c \gamma^{n}(b)$. By our assumptions this yields $b \in \mathbb{C}$, which is a contradiction. Using Theorem 1 we conclude that $R$ is a $\mathbb{Z}$-controlled ring.

\section{A Characterization of $G$-Controlled Strongly $G$-Graded Rings}

In this section we give a characterization of $G$-controlled rings which are strongly $G$-graded (see Theorem 2). We begin by noticing that by Examples 1 and 2 there exist $G$-controlled rings which are not strongly $G$-graded. In many cases, however, $G$-controlness will force the gradation to be strong (see Proposition 3).

Lemma 3 Let $G$ be a group and let $R$ be a $G$-graded ring. If $R$ is $G$-controlled, then the following two assertions hold, for each $g \in G$ :

(i) If $R_{g} R_{g^{-1}}=\{0\}$, then $R_{g^{-1}} R_{g}=\{0\}$;

(ii) If $R_{g} R_{g^{-1}}=R_{e}$, then $R_{g^{-1}} R_{g}=R_{e}$.

Proof We first notice that by Proposition 1(ii), $R_{g}$ is a (non-zero) simple $R_{e}$-sub-bimodule of $R$, for each $g \in G$.

(i): Suppose that $R_{g} R_{g^{-1}}=\{0\}$ holds. Seeking a contradiction, suppose that $R_{g^{-1}} R_{g} \neq$ $\{0\}$. Then $R_{g^{-1}} R_{g}=R_{e}$ and hence $R_{g}=R_{g} R_{e}=R_{g}\left(R_{g^{-1}} R_{g}\right)=\{0\} R_{g}=\{0\}$. By Proposition 1(ii), this is a contradiction.

(ii): This follows from (i).

Recall from [13, Definition 2] that a $G$-graded ring $R$ is said to have a right nondegenerate (resp. left non-degenerate) $G$-gradation if, for each $g \in G$ and each non-zero $x \in R_{g}$, the set $x R_{g^{-1}}$ (resp. $R_{g^{-1}} x$ ) is non-zero.

Proposition 3 Let $G$ be a group and let $R$ be a $G$-graded ring. If $R$ is $G$-controlled, then the following four assertions are equivalent:

(i) $R$ is graded simple;

(ii) $R$ is simple; 
(iii) $R$ is strongly G-graded;

(iv) The G-gradation on $R$ is left (and right) non-degenerate.

Proof (iii) $\Rightarrow$ (iv): This is clear.

(iv) $\Rightarrow$ (ii): Suppose that the $G$-gradation on $R$ is left (and right) non-degenerate. Let $I$ be a non-zero ideal of $R$. It follows from Proposition $1(\mathrm{v})$ that $I$ is graded. Hence, by the assumption and Proposition 1(iii) we conclude that $R_{e} \subseteq I$. Thus, $I=R$.

(ii) $\Rightarrow$ (i): This is clear.

(i) $\Rightarrow$ (iii): Suppose that $R$ is graded simple. Take $g \in G$ and notice that $R_{g}$ is non-zero. By graded simplicity there are some $s, t \in G$ such that $s g t=e$ and $R_{s} R_{g} R_{t}=R_{e}$. From this we get

$$
R_{s} R_{g} R_{t} R_{t^{-1}}=R_{e} R_{t^{-1}}=R_{t^{-1}} \neq\{0\} .
$$

Hence, $R_{t} R_{t^{-1}} \neq\{0\}$ and therefore $R_{t} R_{t^{-1}}=R_{e}$. From this we get $R_{s} R_{g}=R_{t^{-1}}$ and $R_{t^{-1}} R_{t}=R_{e}$, using Lemma 3(ii). From Eq. 1 we get

$$
R_{t} R_{s} R_{g}=R_{t} R_{t^{-1}}=R_{e}
$$

and hence $t s g=e$, i.e. $t s=g^{-1}$. Since $R_{t} R_{s} \subseteq R_{g^{-1}}$, this shows that $R_{g^{-1}} R_{g} \neq\{0\}$ which yields $R_{g^{-1}} R_{g}=R_{e} \ni 1_{R}$. Hence, $R$ is a strongly $G$-graded ring.

Remark 1 If a $G$-controlled ring is e.g. crystalline graded [9] or epsilon-strongly graded [10], then it is necessarily strongly $G$-graded. This follows from Proposition 3 and the fact that both crystalline graded rings and epsilon-strongly graded rings are left (and right) nondegenerate.

Recall that if $T$ is a ring, then a $T$-bimodule $M$ is said to be invertible if there is a $T$ bimodule $N$ such that $M \otimes_{T} N \cong T$ and $N \otimes_{T} M \cong T$. The Picard group of a $\operatorname{ring} T$, denoted by $\operatorname{Pic}(T)$, consists of all equivalence classes of invertible $T$-bimodules and the group operation is given by $\otimes_{T}$. Using that $R$ is strongly $G$-graded, the map $\psi: G \rightarrow$ $\operatorname{Pic}\left(R_{e}\right), g \mapsto\left[R_{g}\right]$ is a group homomorphism (see e.g. [8, Corollary 3.1.2]).

For strongly $G$-graded rings, we record the following observation.

Lemma 4 Let $G$ be a group and let $R$ be a strongly $G$-graded ring. Consider the following assertions:

(i) $C_{R}\left(R_{e}\right)=Z\left(R_{e}\right)$;

(ii) The group homomorphism $\psi: G \rightarrow \operatorname{Pic}\left(R_{e}\right), g \mapsto\left[R_{g}\right]$ is injective.

The following conclusions hold:

(a) (i) implies (ii);

(b) If $R_{e}$ is a simple ring, then (i) holds if and only if (ii) holds;

(c) If $R$ is $G$-controlled, then both ( $i$ ) and (ii) hold.

Proof (a): Suppose that (i) holds. Take $g \in G$ such that $R_{g} \cong R_{e}$. Then there is an $R_{e^{-}}$ bimodule isomorphism $f: R_{e} \rightarrow R_{g}$. We notice that $0 \neq f\left(1_{R}\right) \in R_{g}$. For any $a \in R_{e}$ we have $a f\left(1_{R}\right)=f\left(a 1_{R}\right)=f\left(1_{R} a\right)=f\left(1_{R}\right) a$, showing that $f\left(1_{R}\right) \in C_{R}\left(R_{e}\right)=Z\left(R_{e}\right) \subseteq$ $R_{e}$. Thus, $g=e$. This shows that $\psi$ is injective.

(b): Let $R_{e}$ be a simple ring and suppose that (ii) holds. Notice that $C_{R}\left(R_{e}\right)$ is a $G$-graded subring of $R$. Take $g \in G$ and a non-zero $x_{g} \in C_{R}\left(R_{e}\right) \cap R_{g}$. The set $I=x_{g} R_{g^{-1}} \subseteq R_{e}$ is a non-zero ideal of $R_{e}$. Indeed, by the strong gradation we get $x_{g} R_{g^{-1}} \neq\{0\}$ and from the fact that $R_{g^{-1}}$ is an $R_{e}$-sub-bimodule of $R$ and that $x_{g} \in C_{R}\left(R_{e}\right)$, it follows that $I$ is an ideal of $R_{e}$. By simplicity of $R_{e}$ we get $I=R_{e}$. In particular, there is some $y_{g^{-1}} \in R_{g^{-1}}$ 
such that $x_{g} y_{g^{-1}}=1_{R}$. Symmetrically we get that $R_{g^{-1}} x_{g}=R_{e}$ which yields that $x_{g}$ also has a left inverse. Hence, $x_{g}$ is invertible.

Clearly, $R_{e} x_{g} \subseteq R_{g}$ and $R_{g} y_{g^{-1}} \subseteq R_{e}$. Using that $y_{g^{-1}} x_{g}=1_{R}$ we get $R_{g} \subseteq R_{e} x_{g}$. This shows that $R_{g}=R_{e} x_{g}$.

Notice that $f: R_{e} \rightarrow R_{g}=R_{e} x_{g}, r \mapsto r x_{g}$ is an isomorphism of $R_{e}$-bimodules. By the injectivity of $\psi$ we conclude that $g=e$. Hence, $C_{R}\left(R_{e}\right) \subseteq R_{e}$ which yields $C_{R}\left(R_{e}\right)=$ $Z\left(R_{e}\right)$.

(c): This follows from (a) and Proposition 1(iv).

We are now ready to prove the second main result of this article.

Theorem 2 Let $G$ be a group and let $R$ be a strongly $G$-graded ring. The following three assertions are equivalent:

(i) $R$ is $G$-controlled;

(ii) $R_{g}$ is a simple $R_{e}$-sub-bimodule of $R$, for each $g \in G$, and $C_{R}\left(R_{e}\right)=Z\left(R_{e}\right)$;

(iii) $R_{g}$ is a simple $R_{e}$-sub-bimodule of $R$, for each $g \in G$, and the group homomorphism $\psi: G \rightarrow \operatorname{Pic}\left(R_{e}\right), g \mapsto\left[R_{g}\right]$ is injective.

Proof This follows from Lemma 4 and Theorem 1.

By combining Theorem 2 and Proposition 3 we get the following generalization of [1, Theorem 4(1)].

Corollary 1 If $R$ is a strongly $G$-graded ring such that $R_{g}$ is a simple $R_{e}$-sub-bimodule of $R$, for each $g \in G$, and $C_{R}\left(R_{e}\right)=Z\left(R_{e}\right)$, then $R$ is a simple ring.

The following corollary is an algebraic analogue of [2, Theorem 4.4(i)].

Corollary 2 Let $G$ be a group and let $R$ be a G-crossed product. The following three assertions are equivalent:

(i) $R$ is $G$-controlled;

(ii) $R_{e}$ is a simple ring and $C_{R}\left(R_{e}\right)=Z\left(R_{e}\right)$;

(iii) $R_{e}$ is a simple ring and for every invertible $u_{g} \in R_{g}, g \neq e$, the automorphism of $R_{e}$, defined by $\sigma_{g}(a)=u_{g} a u_{g}^{-1}$, for $a \in R_{e}$, is outer.

Proof Put $R=R_{e} \rtimes_{\sigma}^{\alpha} G$ and let $\left\{u_{g}\right\}_{g \in G}$ be a basis for $R$ as a free left $R_{e}$-module.

(i) $\Rightarrow$ (ii): This follows immediately from Proposition 1.

(ii) $\Rightarrow$ (iii): Suppose that (ii) holds. Take $g \in G$. Suppose that $\sigma_{g}$ is inner, i.e. there is some invertible $v \in R_{e}$ such that $\sigma_{g}(a)=u_{g} a u_{g}^{-1}=v a v^{-1}$ holds for all $a \in R_{e}$. From this we get that $a v^{-1} u_{g}=v^{-1} u_{g} a$ holds for all $a \in R_{e}$. Hence, $v^{-1} u_{g} \in C_{R}\left(R_{e}\right)=Z\left(R_{e}\right) \subseteq R_{e}$ and therefore we must have $g=e$. This shows that (iii) holds.

(iii) $\Rightarrow$ (i): Suppose that (iii) holds. We begin by noticing that $C_{R}\left(R_{e}\right)$ is a $G$-graded subring of $R$. Take $g \in G$. Let $x \in C_{R}\left(R_{e}\right)$ be a non-zero homogeneous element of degree $g$. Then, $x=a u_{g}$ for some $a \in R_{e}$. By definition, $r a u_{g}=a u_{g} r$ for each $r \in R_{e}$. Hence, $r a=a \sigma_{g}(r)$ for each $r \in R_{e}$. From this we get that $a R_{e}=R_{e} a$ is a non-zero two-sided ideal of $R_{e}$. By simplicity of $R_{e}$ we conclude that $a$ is invertible. Hence, $a^{-1} r a=\sigma_{g}(r)$ for each $r \in R_{e}$. In other words, $\sigma_{g}$ is inner. By our assumption we conclude that $g=e$. This shows that $C_{R}\left(R_{e}\right) \subseteq R_{e}$, from which we get $C_{R}\left(R_{e}\right)=Z\left(R_{e}\right)$. Using that $R_{e}$ is a simple ring we conclude that $R_{e} u_{g}$ is a simple $R_{e}$-sub-bimodule of $R$, for each $g \in G$. The desired conclusion now follows directly from Theorem 2 . 
Remark 2 For a skew group ring $A \rtimes_{\sigma} G$, Corollary 2(iii) means that $A$ is a simple ring and that the action of $G$ on $A$ is outer (see e.g [3, 7] or [12]).

Example 3 We shall now apply Corollary 2 to two examples.

(a) Suppose that $L / K$ is a finite Galois extension of fields. Notice that each non-identity element of $G=\operatorname{Gal}(L / K)$ is an outer automorphism of $L$. By taking $\sigma: G \rightarrow \operatorname{Aut}(L)$ to be the natural map, we may form the $G$-controlled skew group ring $L \rtimes_{\sigma} G$.

(b) Recall that each non-identity automorphism of the first Weyl algebra $\mathcal{A}_{1}$ is outer. Hence, by taking any non-identity automorphism $\sigma_{1}: \mathcal{A}_{1} \rightarrow \mathcal{A}_{1}$ we may form a $\mathbb{Z}$-controlled skew group ring $\mathcal{A}_{1} \rtimes_{\sigma} \mathbb{Z}$.

\section{Subrings of Strongly $G$-Graded Rings}

In this section we give a description of certain subrings of $G$-controlled rings. We begin with the following result which generalizes [1, Theorem 4(2)].

Proposition 4 If $R$ is a strongly $G$-graded ring which is $G$-controlled, then there is a oneto-one correspondence between submonoids of $G$ and unital subrings of $R$ containing $R_{e}$ given by

$$
\{\text { Submonoids of } G\} \ni H \stackrel{\phi}{\longmapsto} R_{H}=\oplus_{h \in H} R_{h} .
$$

In particular, if $R$ is a $G$-crossed product, then this occurs if $R_{e}$ is simple and $C_{R}\left(R_{e}\right)=$ $Z\left(R_{e}\right)$.

Proof If $H$ is a submonoid of $G$, then $R_{H}=\oplus_{h \in H} R_{h}$ is a unital subring of $R$, containing $R_{e}$. Hence $\phi$ is well-defined. Moreover, it is clear that if $H_{1} \neq H_{2}$ then $R_{H_{1}} \neq R_{H_{2}}$, and this shows that $\phi$ is injective.

Let $S$ be a unital subring of $R$ containing $R_{e}$. Then $S$ is an $R_{e}$-sub-bimodule of $R$ and hence, by the definition of a $G$-controlled ring, there is a non-empty subset $H \subseteq G$ such that $S=R_{H}$. Take $g, h \in H$. Using that $S$ is a ring and that $R$ is strongly $G$-graded, we have $\{0\} \neq R_{g h}=R_{g} R_{h} \subseteq S$. This shows that $g h \in H$ and hence $H$ is a subsemigroup of $G$. From the fact that $R_{e} \subseteq S$ we get $e \in H$, and hence $H$ is a submonoid of $G$. This shows that $\phi$ is surjective. The last part follows from Corollary 2 .

Corollary 3 Let $G$ be a finite group. If $R$ is a strongly G-graded ring which is $G$-controlled, then there is a one-to-one correspondence between subgroups of $G$ and unital subrings of $R$ containing $R_{e}$ given by

$$
\{\text { Subgroups of } G\} \ni H \stackrel{\phi}{\longmapsto} R_{H}=\oplus_{h \in H} R_{h} .
$$

In particular, if $R$ is a G-crossed product, then this occurs if $R_{e}$ is simple and $C_{R}\left(R_{e}\right)=$ $Z\left(R_{e}\right)$.

Remark 3 Clearly, subrings of $R_{e}$ are also subrings of $R$, but in general they can not be described by the above correspondence. Take e.g. a skew group ring $A \rtimes_{\sigma} G$ and consider the subrings $Z(A)$ respectively $A^{G}=\left\{a \in A \mid \sigma_{g}(a)=a, \forall g \in G\right\}$. Notice that $A^{G}=A$ if and only if $A \rtimes_{\sigma} G$ is a group ring. Hence, in Proposition 4 and Corollary 3 the requirement "subrings of $R$ containing $R_{e}$ " can not be relaxed. 


\section{Simple Strongly $G$-Graded Rings and Some Open Questions}

By Proposition 3, $G$-controlled rings which are strongly $G$-graded are necessarily simple. In this section we shall discuss some known simplicity results for strongly $G$-graded rings and see how they are related to our investigation of $G$-controlness. We will also present some open questions (see Section 6.1).

The following result was shown by Van Oystaeyen (see [15, Theorem 3.4]).

Theorem 3 ([15]) Let $R$ be a strongly G-graded ring such that the morphism $G \rightarrow$ $\operatorname{Pic}\left(R_{e}\right)$, defined by $g \mapsto\left[R_{g}\right]$, is injective. If $R_{e}$ is a simple ring, then $R$ is a simple ring.

Using Lemma 4 we get the following equivalent formulation of Theorem 3.

Proposition 5 Let $R$ be a strongly G-graded ring such that $C_{R}\left(R_{e}\right)=Z\left(R_{e}\right)$ holds. If $R_{e}$ is a simple ring, then $R$ is a simple ring.

It now becomes clear that Van Oystaeyen's result is in fact a generalization of Azumaya's result [1, Theorem 4(1)], from skew group rings by finite groups to general strongly group graded rings. (In fact, it is even more general than Corollary 1.) The following example shows that Proposition 5 does not necessarily hold if we relax the assumption on the strong gradation.

Example 4 If $R$ is not strongly $G$-graded, then assuming $C_{R}\left(R_{e}\right)=Z\left(R_{e}\right)$ and simplicity of $R_{e}$ is not enough to guarantee that $R$ be simple. Indeed, let $F$ be a field and let $\tau$ : $F \rightarrow F$ be a field automorphism of infinite order. We define a (not strongly) $\mathbb{Z}$-graded ring $R=\oplus_{n \in \mathbb{Z}} R_{n}$, with $R_{n}=F u_{n}$ for $n \geq 0$ and $R_{n}=\{0\}$ for $n<0$, whose multiplication is defined by $a u_{n} b u_{m}=a \tau^{n}(b) u_{n+m}$ for $a, b \in F$ and $n, m \in \mathbb{Z}$. Clearly, $F=R_{e}$ is simple and $C_{R}\left(R_{e}\right)=C_{R}(F)=F=Z\left(R_{e}\right)$. However, the ideal generated by $u_{1}$ is proper. Hence, $R$ is not simple. Also notice that $R$ is not $\mathbb{Z}$-controlled.

Remark 4 Let $R$ be a $G$-graded ring.

(a) Suppose that the gradation on $R$ is left (or right) non-degenerate. If $R_{e}$ is a simple ring, then $R$ is graded simple.

(b) $C_{R}\left(R_{e}\right)=Z\left(R_{e}\right)$ is not a necessary condition for simplicity of $R$. To see this, consider e.g. the skew group ring $M_{2}(\mathbb{R}) \rtimes_{\sigma} \mathbb{Z} / 2 \mathbb{Z}$ in [12, Example 4.1].

Recall that a group $G$ is said to be hypercentral if every non-trivial factor group of $G$ has a non-trivial center. Hypercentral groups include e.g. all abelian groups. The following result follows from [5, Theorem 6] and is a partial generalization of Van Oystaeyen's result (Theorem 3).

Proposition 6 Let $G$ be a hypercentral group and let $R$ be a strongly $G$-graded ring. If $R$ is graded simple and $C_{R}\left(R_{e}\right)=Z\left(R_{e}\right)$ holds, then $R$ is a simple ring.

Proof Suppose that $R$ is graded simple and that $C_{R}\left(R_{e}\right)=Z\left(R_{e}\right)$ holds. If we can show that $Z(R)$ is a field, then by [5, Theorem 6] we are done.

Take a non-zero $c \in Z(R) \subseteq C_{R}\left(R_{e}\right)=Z\left(R_{e}\right) \subseteq R_{e}$. Clearly, $c R$ is a non-zero graded ideal of $R$. Hence, by graded simplicity of $R$, we get $c R=R$. From the gradation we 
conclude that $c R_{e}=R_{e}$. In particular, $c$ is invertible in $R_{e}$. One easily verifies that the inverse of $c$ belongs to $Z(R)$. This shows that $Z(R)$ is a field.

Example 5 Consider the matrix ring $R=M_{3}(\mathbb{C})$ equipped with the following gradation by $G=\mathbb{Z} / 2 \mathbb{Z}$.

$$
R_{0}=\left(\begin{array}{lll}
\mathbb{C} & 0 & \mathbb{C} \\
0 & \mathbb{C} & 0 \\
\mathbb{C} & 0 & \mathbb{C}
\end{array}\right) \quad R_{1}=\left(\begin{array}{lll}
0 & \mathbb{C} & 0 \\
\mathbb{C} & 0 & \mathbb{C} \\
0 & \mathbb{C} & 0
\end{array}\right)
$$

A short calculation shows that

$$
C_{R}\left(R_{0}\right)=\left\{\left(\begin{array}{lll}
a & 0 & 0 \\
0 & b & 0 \\
0 & 0 & a
\end{array}\right) \mid a, b \in \mathbb{C}\right\}=Z\left(R_{0}\right) .
$$

Another short calculation shows that $R_{0}$ has two non-trivial ideals;

$$
I=\left(\begin{array}{lll}
0 & 0 & 0 \\
0 & \mathbb{C} & 0 \\
0 & 0 & 0
\end{array}\right) \quad \text { and } \quad J=\left(\begin{array}{lll}
\mathbb{C} & 0 & \mathbb{C} \\
0 & 0 & 0 \\
\mathbb{C} & 0 & \mathbb{C}
\end{array}\right) .
$$

We notice that $R_{1} I R_{1} \subseteq J$ and $R_{1} J R_{1} \subseteq I$. Thus, $R$ is graded simple. Using Proposition 6 we retrieve a well-known fact: the matrix $\operatorname{ring} R=M_{3}(\mathbb{C})$ is simple. Notice, however, that $R=M_{3}(\mathbb{C})$ is not $\mathbb{Z} / 2 \mathbb{Z}$-controlled.

\subsection{Open Questions}

We shall now present some open questions which require further investigation.

Remark 5 Let $R$ be a strongly $G$-graded ring. If $R_{e}$ is a division ring, then it follows almost immediately from the definition of a strongly $G$-graded ring that $R$ is a $G$-crossed product. We notice that, if $G$ is finite, then the assumption on $R_{e}$ can be slightly relaxed. In fact, if $R_{e}$ is a simple and artinian ring, then $R$ is a $G$-crossed product (see e.g. [14, Lemma 1.1]).

It is easy to find examples of $G$-crossed products on which Theorem 3 can be applied. Unfortunately, the literature does not seem to provide any example of a general strongly $G$-graded ring (not a $G$-crossed product) satisfying the conditions of Theorem 3. Based on this, and in light of the above remark, we ask the following question.

Question 1 Let $R$ be a strongly $G$-graded ring for which $R_{e}$ is a simple ring. Is $R$ necessarily a $G$-crossed product?

If the answer to Question 1 is negative, then a natural follow-up question reads as follows.

Question 2 Let $R$ be a strongly $G$-graded ring which is $G$-controlled. Is $R$ necessarily a $G$-crossed product?

We want to know whether Van Oystaeyen's result (Theorem 3) can be generalized to situations when $R_{e}$ is not necessarily simple and ask the following.

Question 3 Let $R$ be a strongly $G$-graded ring. Suppose that $R$ is graded simple and that $C_{R}\left(R_{e}\right)=Z\left(R_{e}\right)$ holds. Is $R$ necessarily simple? 
Remark 6 Notice that Question 3 is known to have an affirmative answer in (at least) the following three cases:

1. $R_{e}$ is simple (see Proposition 5);

2. $R_{e}$ is commutative (see [11, Theorem 6.6]);

3. $G$ is a hypercentral group (see Proposition 6).

Acknowledgements The author would like to thank an anonymous referee for valuable comments on the manuscript and for kindly having provided Example 1.

Open Access This article is distributed under the terms of the Creative Commons Attribution 4.0 International License (http://creativecommons.org/licenses/by/4.0/), which permits unrestricted use, distribution, and reproduction in any medium, provided you give appropriate credit to the original author(s) and the source, provide a link to the Creative Commons license, and indicate if changes were made.

\section{References}

1. Azumaya, G.: New foundation of the theory of simple rings. Proc. Japan Acad. 22(11), 325-332 (1946)

2. Cameron, J., Smith, R.R.: Bimodules in crossed products of von Neumann algebras. Adv. Math. 274, 539-561 (2015)

3. Crow, K.: Simple regular skew group rings. J. Algebra Appl. 4(2), 127-137 (2005)

4. Dade, E.C.: Group-graded rings and modules. Math. Z. 174, 241-262 (1980)

5. Jespers, E.: Simple graded rings. Comm. Algebra 21(7), 2437-2444 (1993)

6. Karpilovsky, G.: The Algebraic Structure of Crossed Products, North-Holland Mathematics Studies, 142. Notas De Matemática [Mathematical Notes], vol. 118, p. x+348. North-Holland Publishing Co., Amsterdam (1987). ISBN: 0-444-70239-3

7. Montgomery, S.: Fixed Rings of Finite Automorphism Groups of Associative Rings, Lecture Notes in Mathematics, vol. 818, p. vii+126. Springer, Berlin (1980). ISBN: 3-540-10232-9

8. Nastasescu, C., Van Oystaeyen, F.: Methods of Graded Rings, Lecture Notes in Mathematics, vol. 1836, p. xiv+304. Springer, Berlin (2004). ISBN: 3-540-20746-5

9. Nauwelaerts, E., Van Oystaeyen, F.: Introducing crystalline graded algebras. Algebr. Represent. Theory 11(2), 133-148 (2008)

10. Nystedt, P., Öinert, J., Pinedo, H.: Epsilon-strongly graded rings, separability and semisimplicity, arXiv:1606.07592 [math.RA]

11. Öinert, J.: Simple Group Graded Rings and Maximal Commutativity, Operator Structures and Dynamical Systems (Leiden, NL, 2008), 159-175, Contemp. Math, vol. 503. Amer. Math. Soc., Providence, RI (2009)

12. Öinert, J.: Simplicity of skew group rings of abelian groups. Comm. Algebra 42(2), 831-841 (2014)

13. Öinert, J., Lundström, P.: The ideal intersection property for groupoid graded rings. Comm. Algebra 40(5), 1860-1871 (2012)

14. Schmid, P.: Clifford theory of simple modules. J. Algebra 119(1), 185-212 (1988)

15. Van Oystaeyen, F.: On Clifford systems and generalized crossed products. J. Algebra 87(2), 396-415 (1984) 